Research Article

\title{
Study on Size-Dependent Young's Modulus of a Silicon Nanobeam by Molecular Dynamics Simulation
}

\author{
H. Yu, C. Sun, W. W. Zhang, S. Y. Lei, and Q. A. Huang \\ Key Laboratory of MEMS of Ministry of Education, Southeast University, Nanjing 210096, China \\ Correspondence should be addressed to H. Yu; h_yu@seu.edu.cn
}

Received 26 October 2012; Revised 7 January 2013; Accepted 21 January 2013

Academic Editor: Zhenhui Kang

Copyright (C) $2013 \mathrm{H}$. Yu et al. This is an open access article distributed under the Creative Commons Attribution License, which permits unrestricted use, distribution, and reproduction in any medium, provided the original work is properly cited.

\begin{abstract}
Young's modulus of a silicon nanobeam with a rectangular cross-section is studied by molecular dynamics method. Dynamic simulations are performed for doubly clamped silicon nanobeams with lengths ranging from 4.888 to $12.491 \mathrm{~nm}$ and cros-sections ranging from $1.22 \mathrm{~nm} \times 1.22 \mathrm{~nm}$ to $3.39 \mathrm{~nm} \times 3.39 \mathrm{~nm}$. The results show that Young's moduli of such small silicon nanobeams are much higher than the value of Young's modulus for bulk silicon. Moreover, the resonant frequency and Young's modulus of the Si nanobeam are strongly dependent not only on the size of the nanobeam but also on surface effects. Young's modulus increases significantly with the decreasing of the thickness of the silicon nanobeam. This result qualitatively agrees with one of the conclusions based on a semicontinuum model, in which the surface relaxation and the surface tension were taken into consideration. The impacts of the surface reconstruction with $(2 \times 1)$ dimmers on the resonant frequency and Young's modulus are studied in this paper too. It is shown that the surface reconstruction makes the silicon nanobeam stiffer than the one without the surface reconstruction, resulting in a higher resonant frequency and a larger Young's modulus.
\end{abstract}

\section{Introduction}

Nanoelectromechanical systems (NEMS) have been found in many important applications, such as ultrasensitive mass sensing [1-3], ultralow-power radio frequency (RF) signal generation $[4,5]$, parametric feedback oscillating [6], and switch $[7,8]$. In these applications, silicon-based nanowires and nanobeams with very small dimensions and high performances played important roles. Nanoscale cantilevers and doubly clamped beams were two basic types of beams, and were used as resonators in these applications $[3,4,9,10]$, where they were oscillating in $\mathrm{MHz}$ or even in GHz. From the view point of long-term reliability of these resonators, the vibration behavior and mechanical properties, such as Young's modulus, are very important and should be paid much attention by researchers.

A number of researches have been done on the vibration and elastic characteristics of nanostructures including experiments, theoretical analysis, and atomistic or molecular dynamics simulations. In some experimental researches, equations based on continuum assumption were applied to study the resonance of silicon nanobeams/nanowires and the relationship between the resonant frequency and the Young's modulus $[4,11]$. A 3D finite element model taking into account the surface effect was proposed by Feng et al. [12], in which Young's modulus and the resonant frequency are both found to decrease significantly with the decreasing of the diameter of silicon nanowires for fixed/free boundary condition when the diameter is less than $100 \mathrm{~nm}$, while they slightly decrease for fixed/fixed boundary condition. However, some researchers pointed out that classical theories or computational design tools, which are based on continuum assumptions, may not be directly applicable for nanostructures because of their low dimensions. Several approaches have been proposed to extend classical continuum theories to nanostructured materials by considering the discrete nature of nanomaterials. For example, Sun and Zhang developed a semicontinuum model to calculate Young's modulus of the ultrathin film with a simple cubic lattice [13]. They found that the value of Young's modulus depends on the number of atom layers in the thickness direction; that is, Young's modulus decreases with the decreasing of the number of atom layers and approaches the bulk value as the number of atom layers increases. Later, a lattice model was developed by 
Guo and Zhao to investigate the size-dependent properties of a nanofilm with a simple cubic lattice, which took the surface relaxation into consideration [14]. They concluded that Young's modulus depends not only on the thickness of parameter but also on the surface relaxation. The value of Young's modulus can increase with the decreasing of the number of atomic layers under some surface relaxation conditions. Based on the semicontinuum theory and the Keating model, which was especially well suited to describing IV semiconductor crystal, we studied the size and temperature dependence of Young's modulus of a silicon nanoplate [15] and the influence of surface effects on the mechanical properties of silicon nanobeams [16]. It was predicted that the effective elastic modulus, $E_{\text {eff }}$, of a bending silicon nanobeam depends on the competition between the surface relaxation coefficient $k_{i}$ and the surface tension $\gamma$. When the number of the cell layers is less than 15 , the surface tension will dominate the variation of the effective elastic modulus, and it will be increasing dramatically with the decreasing of the thickness of the silicon nanobeam when $\gamma>0$. Rudd et al. performed classical molecular dynamics simulations to study the elastic behavior of silicon nanowires based on the Stillinger-Weber (SW) potential $[17,18]$. In 2008, they studied the mechanics of silicon nanowires again by performing first-principles density functional theory calculations and compared their results to prior results from MD methods. They concluded that for hydrogen-passivated silicon $\langle 001\rangle$ nanowires Young's modulus decreases with the decreasing of the diameter of nanowires [19]. By performing the molecular dynamics simulations with the use of the Tersoff potential, Park et al. simulated the flexural and the longitudinal vibrations of silicon nanocantilevers and deduced the elastic modulus and Young's modulus for the nanothick silicon cantilever from the natural frequency equation based on the continuum theory $[20,21]$. Their conclusion was that Young's modulus decreases as the thickness of the cantilever is decreased.

In this paper, the resonant behavior of doubly clamped silicon nanobeams is first studied by using a specific computer module, Forcite, which is based on the molecular dynamics method. The value of Young's modulus of the silicon nanobeam is then deduced. It is found that the variation of Young's modulus of the silicon nanowire is contrary to the results from Park et al. [20, 21] and Rudd and Lee [19], but it is in agreement with our prior results based on the semicontinuum model [16], which took the surface relaxation and the surface stress into consideration.

\section{Simulation Model}

The prototype under study is a doubly clamped silicon $\langle 001\rangle$ nanobeam with a rectangular cross-section. The simulation model of the silicon nanobeam is built by the software as described in the reference [22]. The orientation and the crosssection of the silicon nanobeam are redepicted here, as shown as in Figure 1(a). A rectangular coordinate system is used with $x$ - and $z$-axes along the length and width directions, respectively, and $y$-axis along the thickness direction perpendicular to the (001) surface of the silicon nanowire. Periodic

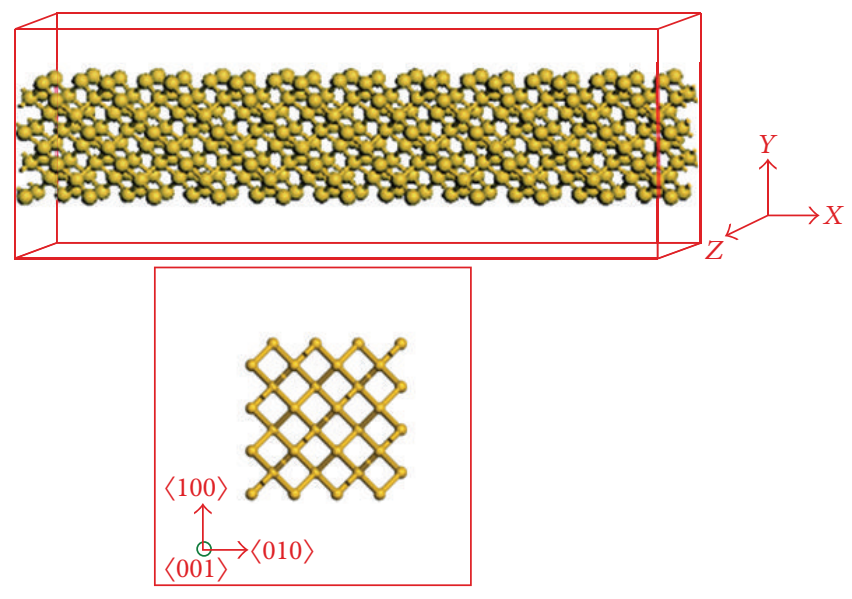

(a)

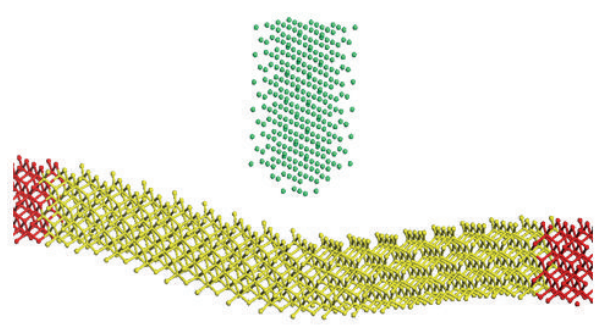

(b)

FIgURE 1: A schematic of a doubly clamped silicon nanobeam (a) orientation and cross-section and (b) an AFM probe (green atoms) and the silicon nanobeam after structure optimization.

boundary condition is used in the longitudinal direction of the Si nanobeam, and lateral surfaces are under free boundary conditions in vacuum.

To actuate the beam, one silicon AFM probe is built to be close to the beam. This is different from Park's simulation model, where the clamped-free cantilever was induced with flexural or longitudinal vibration by simulating suitable initial conditions [20,21]. After structure optimization, the nanobeam becomes bending since Van der Waals force exists between the AFM and the nanobeam, as shown as in Figure 1(b), where atoms (red atoms) at two ends of the nanobeam are clamped. Because of the initial bending, the nanobeam will vibrate freely after the AFM is removed.

The simulation has been performed at the average temperature of $298 \mathrm{~K}$ with a time step of $1 \mathrm{fs}$. Condensedphase optimized molecular potential for atomistic simulation studies (COMPASS) force field is used when the simulation is performed.

\section{Results and Discussion}

The kinetic and the potential energies of the silicon nanobeam are monitored while the nanobeam is vibrating. The frequency response of the silicon nanobeam can be obtained by performing FFT of the kinetic energy or the potential 


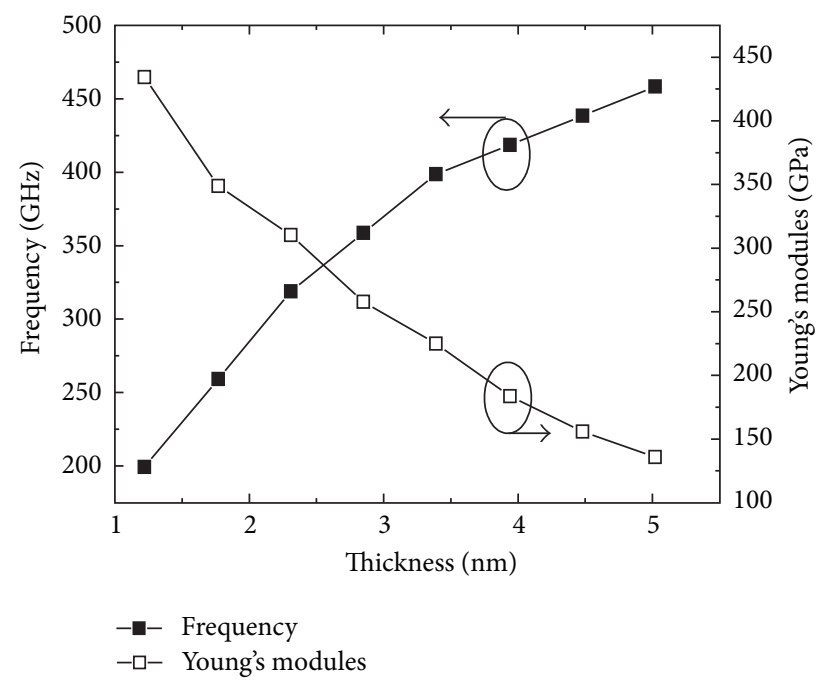

Figure 2: Resonant frequency and Young's modulus as functions of nanobeam thickness.

energy. In Figure 2, the resonant frequency of the nanobeam is shown as a function of the thickness of the nanobeam, where all nanobeams have the same length of $9.232 \mathrm{~nm}$ and the same width of $2.85 \mathrm{~nm}$. It is no surprise that the resonant frequency decreases with the decreasing of the thickness of the beam. However, it should be noticed that the vibration frequencies of such small silicon beams can be in the range of hundreds of $\mathrm{GHz}$, much higher than those of the macroscopic ones. This result means that silicon nanobeams may have promising application in ultrahigh speed wireless communications.

As mentioned above, many experimental and theoretical methods have been developed to evaluate the Young's modulus of nano films and beams. Among these methods, the resonant frequencies of ultrathin silicon cantilevers were used to calculate Young's modulus by Park et al. [20, 21]. As Park did, now we deduce Young's modulus of the silicon nanobeam from our simulation results and Euler-Bernoulli theory. For a doubly clamped Euler-Bernoulli beam, the frequency of the first order resonant mode can be written as following equation [23]:

$$
\omega_{0}=\sqrt{\frac{E I}{m l^{4}}},
$$

where, $\omega_{0}$ is angular frequency of the first resonant mode, $E$ is Young's modulus, $m$ is mass per unit length, $m=\rho A, \rho$ is density of the beam, $A$ is area of the cross-section, and $l$ and $I$ are beam length and mass moment of inertia, respectively. Equation (1) can be rewritten as

$$
f=\frac{i^{2} \pi t}{2 l^{2}} \sqrt{\frac{E}{12 \rho}}
$$

for one doubly clamped beam with a rectangular crosssection, where $f$ is the resonant frequency and $t$ is thickness of the beam. For the first-order resonant mode, $i \approx 1.5$. Based on (2), we can deduce the Young's modulus of the

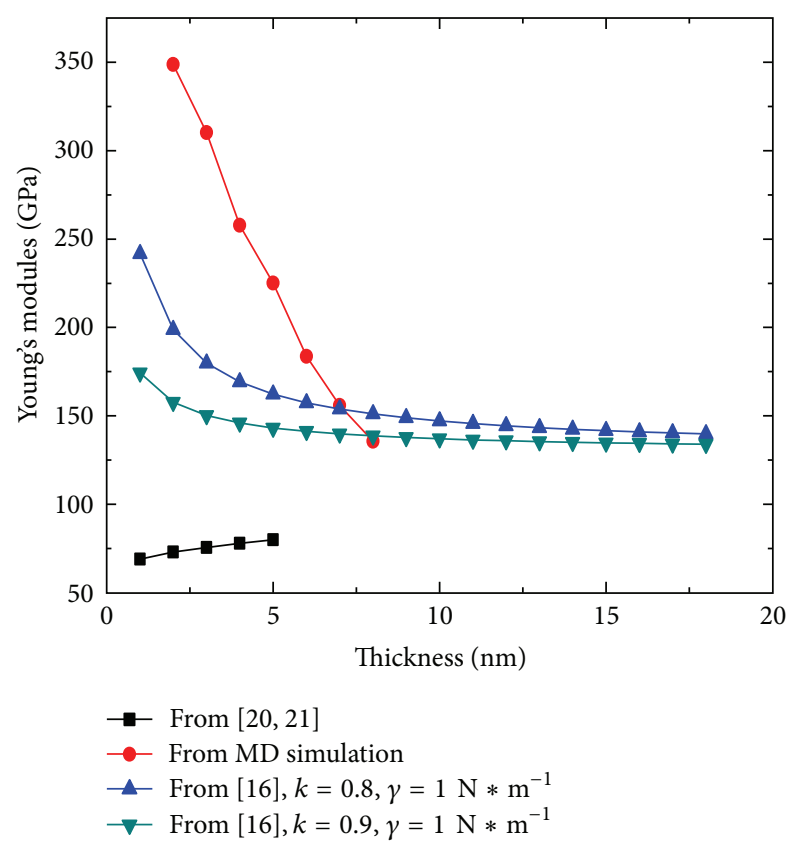

FIGURE 3: Young's modulus as a function of the thickness of the silicon nanobeam based on different models.

doubly clamped silicon nanobeam. The calculated values of the Young's modulus for silicon nanobeams with different thicknesses are shown in Figure 2.

It can be seen from Figure 2 that Young's modulus increases dramatically when the thickness of the nanobeam is decreasing. This result is contrary to that obtained by Park et al. [20, 21]. However, this trend is qualitatively in agreement with the result which is based on the semicontinuum model with surface relaxation coefficient, $k_{i}<1$, and surface stress, $\gamma>0$ [16]. Our simulation results, the results from [16], and results from [20,21] are compared as shown as in Figure 3.

In our simulations, the doubly clamped nanobeam is formed by cleaving silicon surfaces, leaving two dangling bonds of each silicon atom on (001) ideal surface. The termination of the lattice periodicity in the surface normal direction will lead to the imperfection of the coordination numbers (CNs) of a surface atom, which can make the remaining bonds of this lower-coordinated surface atom relaxation [14]. The $\mathrm{CN}$-imperfection-induced bond relaxation can result in that the bond length becomes longer or shorter than the ideal bond length. In our simulation, the structure optimization makes the bond lengths shrink within several layers, which are close to the surface. It means that the contraction surface relaxation occurs after the structure optimization. The effect of contraction surface relaxation makes the nanowire stiffer than the one without relaxation on the surfaces. Furthermore, as predicted as in [16], the bending of the doubly clamped beam will cause the surface tension, which can impact on Young's modulus strongly, and will dominate the variation of the effective elastic modulus when 
the thickness of the nanobeam is less than $8 \mathrm{~nm}$ [16]. As the thickness is decreasing, the combination effect of the surface relaxation and the surface tension becomes stronger, resulting in Young's modulus of the silicon nanobeam increasing with the decreasing of the thickness of the nanobeam. Of course, it should be noticed that there is a deviation between the results from our simulations and those from [16]. We are trying to find out the reason and the explanation.

Besides the surface relaxation and the surface tension, surface reconstructions can also strongly impact the elasticity of a silicon nanoplate $[24,25]$. It has been indicated that there are three kinds of reconstructions on silicon (001) surface. They are symmetrical $(2 \times 1), p(2 \times 2)$, and $c(4 \times 2)$ surface reconstructions. Sadeghian et al. studied the impact of applied deformation on surface reconstruction and elastic behavior of silicon nanobeams [26]. They found that for symmetrical $(2 \times 1)$ reconstruction the applied stain within the stability range is smaller, and the total energy of the nanobeam with this kind of reconstruction is the lowest. If the applied external uniaxial tensile strain increases, the stability range of any relevant reconstructions changes, thus, the surface region undergoes a series of reconstructions. Therefore, Young's modulus of silicon nanobeams under different surface reconstructions will be definitely different and may undergo a series of changes. To simplify the simulations, the $(2 \times 1)$ dimmer reconstruction is only considered on four of the lateral surfaces of the silicon nanobeam in this paper to study the effects of the surface reconstruction on the vibration frequency and Young's modulus. Figure 4 shows the resonant frequency and Young's modulus as functions of cross-sections with and without the reconstruction. It can be seen that the frequency under the surface reconstruction is higher than that without the reconstruction. Because of the surface reconstruction, the nanobeam becomes stiffer, and Young's modulus becomes larger, resulting in the higher resonant frequency. The size of the cross-section is smaller and the changes of resonant frequency and Young's modulus are greater, because the surface to volume ratio (SVR) is higher for the nanobeam with a smaller cross-section and the surface effect is stronger.

The results above are based on the simulations for silicon nanobeams with rectangular cross-sections. It is shown that the surface effects on Young's modulus become stronger for the nanobeam with a smaller dimension (a higher SVR). Since the SVR is strongly dependent on the shape of the crosssection too, it can be predicted that Young's modulus and other properties of silicon nanobeams with other shapes of the cross section will definitely have different values. Zhang et al. and $\mathrm{Xu}$ and Servati studied the changing of cohesive energies and electrical properties of silicon nanowires with different shapes of cross-sections as the SVR increases [2729]. So far, however, the influences of the shape of the crosssection on Young's modulus and the vibration behavior of silicon nanobeams have not been reported yet. Furthermore, reconstructions on lateral surfaces of silicon nanobeams with other shapes of cross-sections will become more complex and must be considered very carefully. The combination effect of the shape of cross-sections and the surface reconstruction on Young's modulus of silicon nanobeams is an interesting

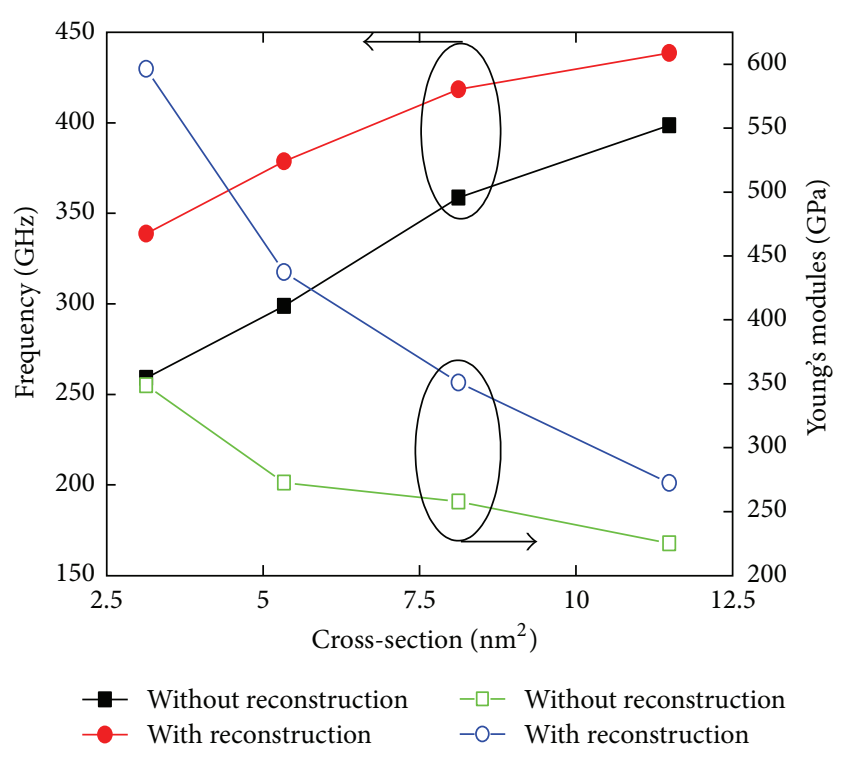

FIGURE 4: Resonant frequencies and Young's moduli of silicon nanobeams with different cross-sections, with and without $(2 \times 1)$ dimmer reconstruction on lateral surfaces.

but very complex issue. This study is being performed by our group.

\section{Conclusions}

Young's modulus of the silicon nanobeam is studied by molecular dynamics. Since the dimensions of the silicon nanobeams are so small that the surface effects cannot be ignored. The resonant frequency of the silicon nanobeam is as high as hundreds of GHz, and Young's modulus is higher than the value of Young's modulus for bulk silicon. Young's modulus of the silicon nanobeam is increasing significantly with the decreasing of thickness of the nanobeam. The resonant frequency and Young's modulus of the doubly clamped silicon nanobeam strongly depend on the thickness of the nanobeam and the surface effects, such as the surface relaxation, the surface tension, and the surface reconstruction. The variation trend of Young's modulus is different from the results from some researches, but is qualitatively in agreement with the results based on the semicontinuum approach, which took the surface relaxation and the surface tension into consideration. The influence of surface effects is stronger on the vibration behavior and Young's modulus when the size of the silicon nanobeam is smaller.

\section{Acknowledgment}

This study is partially supported by the National Science Foundation of China (Grant no. 61001044).

\section{References}

[1] Y. T. Yang, C. Callegari, X. L. Feng, K. L. Ekinci, and M. L. Roukes, "Zeptogram-scale nanomechanical mass sensing," Nano Letters, vol. 6, no. 4, pp. 583-586, 2006. 
[2] Y. T. Yang, C. Callegari, X. L. Feng, and M. L. Roukes, "Surface adsorbate fluctuations and noise in nanoelectromechanical systems," Nano Letters, vol. 11, no. 4, pp. 1753-1759, 2011.

[3] I. Bargatin, E. B. Myers, J. S. Aldridge et al., "Large-scale integration of nanoelectromechanical systems for gas sensing applications," Nano Letters, vol. 12, pp. 1269-1274, 2012.

[4] X. L. Feng, R. He, P. Yang, and M. L. Roukes, "Very high frequency silicon nanowire electromechanical resonators," Nano Letters, vol. 7, no. 7, pp. 1953-1959, 2007.

[5] N. Liu, F. Giesen, M. Belov et al., "Time-domain control of ultrahigh-frequency nanomechanical systems," Nature Nanotechnology, vol. 3, no. 12, pp. 715-719, 2008.

[6] L. G. Villanueva, R. B. Karabalin, M. H. Matheny, E. Kenig, M. C. Cross, and M. L. Roukes, "A nanoscale parametric feedback oscillator," Nano Letters, vol. 11, pp. 5054-5059, 2011.

[7] E. F. Arkan, D. Sacchetto, I. Yildiz, Y. Leblebici, and B. E. Alaca, "Monolithic integration of Si nanowires with metallic electrodes: NEMS resonator and switchapplications," Journal of Micromechanics and Microengineering, vol. 21, Article ID 125018, 9 pages, 2011.

[8] X. L. Feng, M. H. Matheny, C. A. Zorman, M. Mehregany, and M. L. Roukes, "Low voltage nanoelectromechanical switches based on silicon carbide nanowires," Nano Letters, vol. 10, pp. 2891-2896, 2010.

[9] Y. Yang, X. Xia, X. Gan, P. Xu, H. Yu, and X. Li, "Nano-thick resonant cantilevers with a novel specific reaction-induced frequency-increase effect for ultra-sensitive chemical detection," Journal of Micromechanics and Microengineering, vol. 20, no. 5, Article ID 055022, 2010.

[10] A. K. Naik, M. S. Hanay, W. K. Hiebert, X. L. Feng, and M. L. Roukes, "Towards single-molecule nanomechanical mass spectrometry," Nature Nanotechnology, vol. 4, no. 7, pp. 445-450, 2009.

[11] M. Belov, N. J. Quitoriano, S. Sharma, W. K. Hiebert, T. I. Kamins, and S. Evoy, "Mechanical resonance of clamped silicon nanowires measured by optical interferometry," Journal of Applied Physics, vol. 103, Article ID 074304, 7 pages, 2008.

[12] Y. K. Feng, Y. L. Liu, and B. Wang, "Finite element analysis of resonant properties of silicon nanowires with consideration of surface effects," Acta Mechanica, vol. 217, pp. 149-155, 2011.

[13] C. T. Sun and H. Zhang, "Size-dependent elastic moduli of platelike nanomaterials," Journal of Applied Physics, vol. 93, no. 2, pp. 1212-1218, 2003.

[14] J. G. Guo and Y. P. Zhao, "The size-dependent elastic properties of nanofilms with surface effects," Journal of Applied Physics, vol. 98, Article ID 074306, 11 pages, 2005.

[15] J. Wang, Q. A. Huang, and H. Yu, "Size and temperature dependence of Young's modulus of a silicon nano-plate," Journal of Physics D, vol. 41, Article ID 165406, 5 pages, 2008.

[16] J. H. Zhang, Q. A. Huang, H. Yu, and J. Wang, "The influence of surface effects on size-dependent mechanical properties of silicon nanobeams at finite temperature," Journal of Physics D, vol. 42, Article ID 045409, 6 pages, 2009.

[17] R. E. Rudd and J. Q. Broughton, "Atomistic simulation of MEMS resonators through the coupling of length scales," Journal of Modeling and Simulation of Microsystems, vol. 1, no. 1, pp. 2938, 1999.

[18] R. E. Rudd, "Coarse-grained molecular dynamics for computer modeling of nanomechanical systems," International Journal on Multiscale Computational Engineering, vol. 2, p. 203, 2004.
[19] R. E. Rudd and B. Lee, "Mechanics of silicon nanowires: sizedependent elasticity from first principles," Molecular Simulation, vol. 34, no. 1, pp. 1-8, 2008.

[20] S. H. Park, J. S. Kim, J. H. Park, J. S. Lee, Y. K. Choi, and O. M. Kwon, "Molecular dynamics study on size-dependent elastic properties of silicon nanocantilevers," Thin Solid Films, vol. 492, no. 1-2, pp. 285-289, 2005.

[21] J. S. Kim, S. H. Park, J. H. Park, and J. S. Lee, "Molecular dynamics simulation of elastic properties of silicon nanocantilevers," Nanoscale and Microscale Thermophysical Engineering, vol. 10, no. 1, pp. 55-65, 2006.

[22] H. Yu, W. W. Zhang, S. Y. Lei, L. B. Lu, C. Sun, and Q. A. Huang, "Study on vibration behavior of doubly-clamped silicon nanowires by molecular dynamics," Journal of Nanomaterials, vol. 2012, Article ID 342329, 5 pages, 2012.

[23] W. J. Bottega, Engineering Vibrations, CRC Press; Taylor \& Francis, Boca Raton, Fla, USA, 2006.

[24] H. W. Shim, L. G. Zhou, and H. C. Huang, "Nanoplate elasticity under surface reconstruction," Applied Physics Letters, vol. 86, Article ID 151912, 3 pages, 2005.

[25] L. G. Zhou and H. Huang, "Are surfaces elastically softer or stiffer?” Applied Physics Letters, vol. 84, no. 11, pp. 1940-1942, 2004.

[26] H. Sadeghian, J. F. L. Goosen, A. Bossche, B. J. Thijsse, and F. van Keulen, "Surface reconstruction and elastic behavior of silicon nanobeams: the impact of applied deformation," Thin Solid Films, vol. 518, no. 12, pp. 3273-3275, 2010.

[27] R. Q. Zhang, Y. Lifshitz, D. D. D. Ma et al., "Structures and energetics of hydrogen-terminated silicon nanowire surfaces," Journal of Chemical Physics, vol. 123, no. 14, Article ID 144703, 5 pages, 2005.

[28] W. Fan, L. F. Li, K. P. Dou, and R. Q. Zhang, "First principles calculations of transport properties in Si nanowires: the role of crystal orientation," Solid State Communications, vol. 152, pp. 2008-2012, 2012.

[29] X. Xu and P. Servati, "Facet-dependent electronic properties of hexagonal silicon nanowires under progressive hydroxylation and surface reconstruction," Nano Letters, vol. 9, no. 5, pp. 19992004, 2009. 

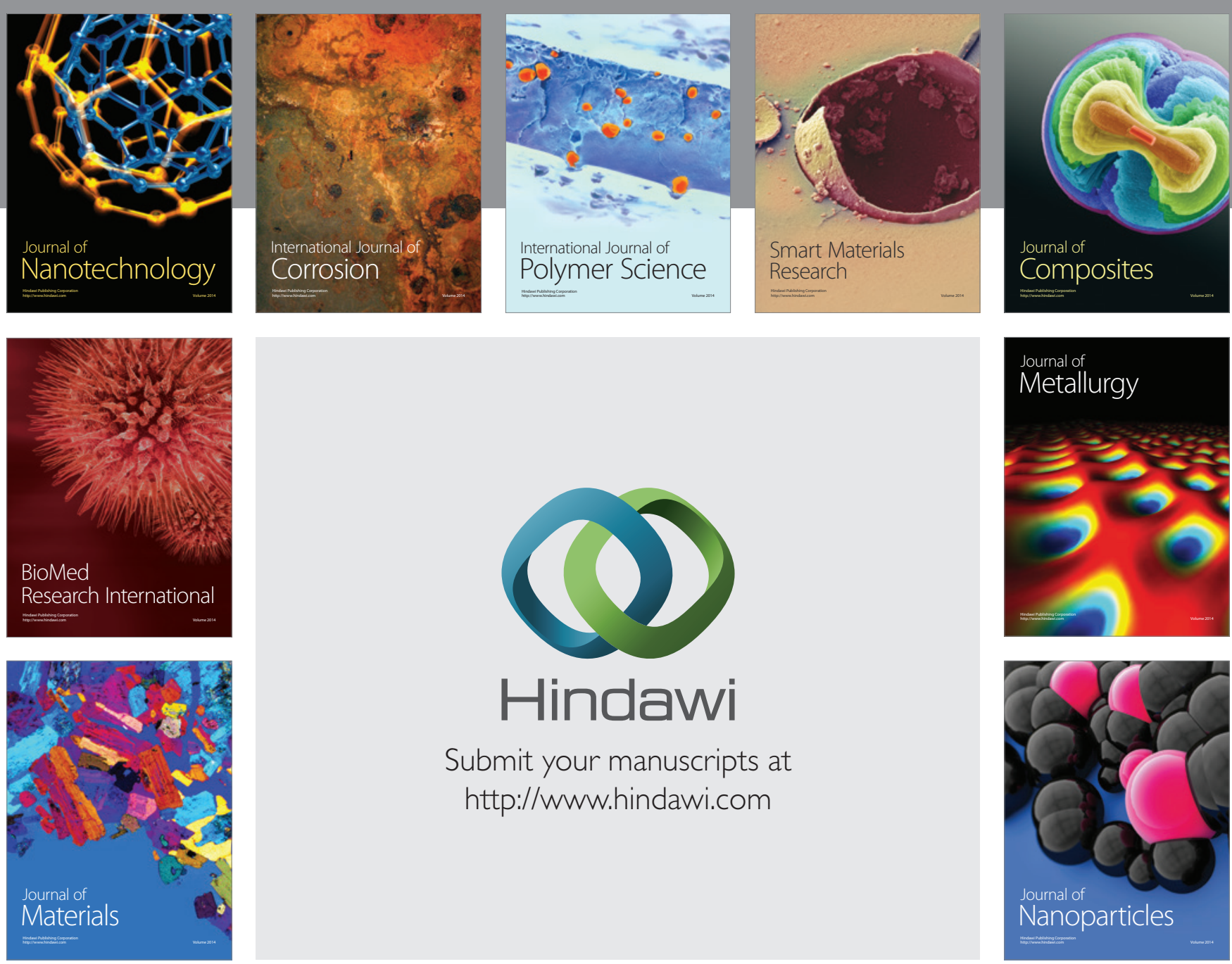

Submit your manuscripts at http://www.hindawi.com
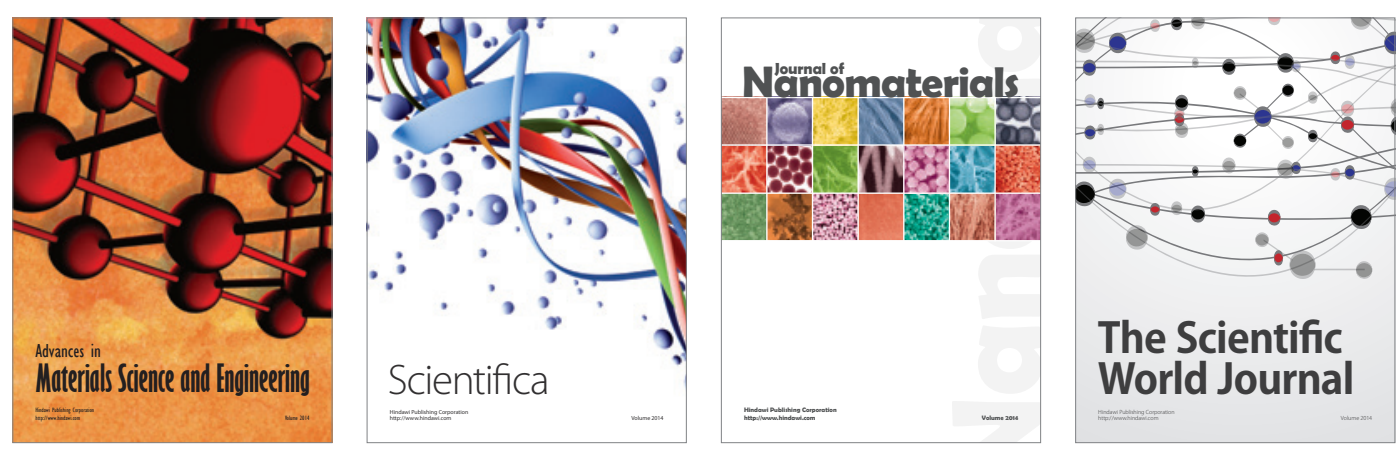

\section{The Scientific World Journal}
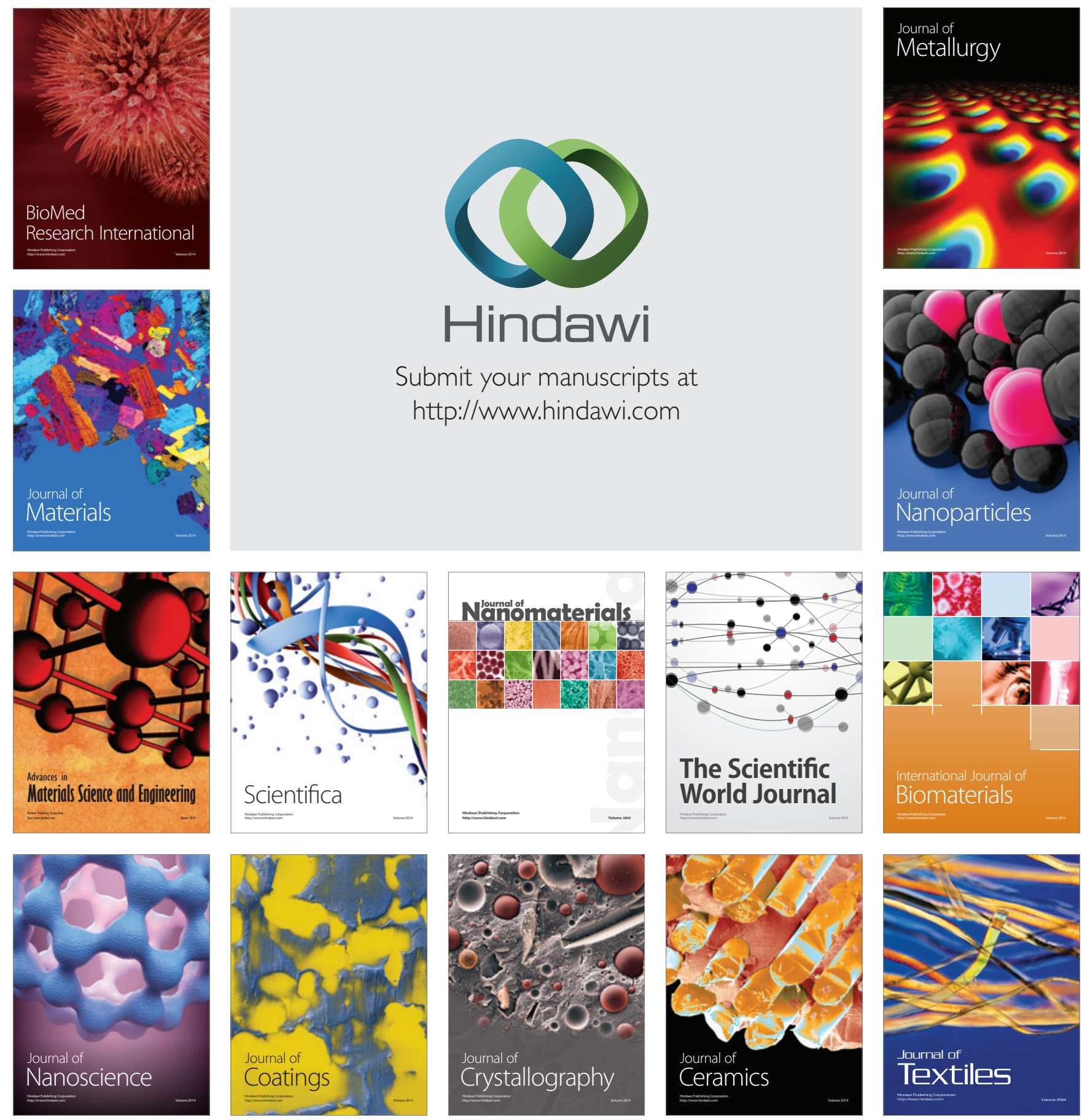